\title{
Artificial Neural Network to Determine Dynamic Effect in Capillary Pressure Relationship for Two-Phase Flow in Porous Media with Micro-Heterogeneities
}

\author{
Diganta B. Das • Thanit Thirakulchaya • Lipika Deka • \\ Navraj S. Hanspal
}

Received: 25 July 2014 / Accepted: 27 October 2014 /Published online: 8 November 2014

C The Author(s) 2014. This article is published with open access at Springerlink.com

\begin{abstract}
An artificial neural network (ANN) is presented for computing a parameter of dynamic two-phase flow in porous media with water as wetting phase, namely, dynamic coefficient $(\tau)$, by considering micro-heterogeneity in porous media as a key parameter. $\tau$ quantifies the dependence of time derivative of water saturation on the capillary pressures and indicates the rates at which a two-phase flow system may reach flow equilibrium. Therefore, $\tau$ is of importance in the study of dynamic two-phase flow in porous media. An attempt has been made in this work to reduce computational and experimental effort by developing and applying an ANN which can predict the dynamic coefficient through the "learning" from available data. The data employed for testing and training the ANN have been obtained from computational flow physics-based studies. Six input parameters have been used for the training, performance testing and validation of the ANN which include water saturation, intensity of heterogeneity, average permeability depending on this intensity, fluid density ratio, fluid viscosity ratio and temperature. It is found that a 15 neuron, single hidden layer ANN can characterize the relationship between media heterogeneity and dynamic coefficient and it ensures a reliable prediction of the dynamic coefficient as a function of water saturation.
\end{abstract}

Keywords Artificial neural network (ANN) - Two phase flow · Porous media · Dynamic coefficient · Dynamic capillary pressure $\cdot$ Porous medium heterogeneity · Micro-heterogeneity

D. B. Das $(\bowtie) \cdot$ T. Thirakulchaya

Chemical Engineering Department, Loughborough University, Loughborough LE11 3TU, UK e-mail: D.B.Das@lboro.ac.uk

L. Deka

School of Civil and Building Engineering, Loughborough University, Loughborough LE11 3TU, UK

N. S. Hanspal

Ansys, Inc., 2645 Zanker Road, San Jose, CA 95134, USA 


\section{Introduction}

Mathematical models of two-phase (e.g., oil-water) flow in porous medium require equations for conservation of fluids' mass and momentum in conjunction with appropriate constitutive equations for capillary pressure $\left(\mathrm{P}^{\mathrm{c}}\right)$-saturation $\left(\mathrm{S}_{\mathrm{w}}\right)$-relative permeability $\left(\mathrm{K}_{\mathrm{r}}\right)$ relationships (Ataie-Ashtiani et al. 2003; Das and Mirzaei 2013). In these models, the capillary pressure is commonly described by formulation such as the Brooks-Corey (Brooks and Corey 1964) or van Genuchten (van Genuchten 1980) relationship where the capillary pressure is defined as an empirical function of water saturation as shown in Eq. (1):

$$
\mathrm{P}_{\mathrm{nw}}-\mathrm{P}_{\mathrm{w}}=\mathrm{P}^{\mathrm{c}}\left(\mathrm{S}_{\mathrm{w}}\right)
$$

Here, $\mathrm{P}_{\mathrm{nw}}, \mathrm{P}_{\mathrm{w}}, \mathrm{P}^{\mathrm{c}}\left(\mathrm{S}_{\mathrm{w}}\right)$ are the average non-wetting fluid (e.g., oil) pressure, wetting fluid (e.g., water) pressure, and capillary pressure at equilibrium condition, i.e., when the rate of change of saturation in the domain is zero $\left(\mathrm{dS}_{\mathrm{w}} / \mathrm{dt}=0\right)$, respectively. As discussed extensively in the literature, the properties of the two-phase system (e.g., interfacial tension, capillary pressure, density and viscosity of each fluid phase) and porous medium (e.g., porosity, permeability, pore size distribution, heterogeneity) are important in determining the flow behavior and, hence, the $\mathrm{P}^{\mathrm{c}}-\mathrm{S}_{\mathrm{w}}$ relationships (Bear 1972; Corey 1994; Das et al. 2004; Das et al. 2007; Das and Mirzaei 2012, 2013; Abidoye and Das 2014). In most cases, empirical data for the $P^{c}-S_{w}$ relationships are obtained by means of laboratory experiments carried out on porous samples that are up to 10-12 cm long, and it is often assumed that the porous sample is homogeneous at that scale. However, it is known that even at the sub-sample scale some heterogeneities (e.g., micro-scale heterogeneity) may exist. These heterogeneities have specific two-phase flow properties and affect the $\mathrm{P}^{\mathrm{c}}-\mathrm{S}_{\mathrm{w}}$ relationships (Das et al. 2004; Das and Mirzaei 2013). To quantify how the heterogeneities affect the $\mathrm{P}^{\mathrm{c}}-\mathrm{S}_{\mathrm{w}}$ relationships, a number of studies have been conducted (Ataie-Ashtiani et al. 2002; Das et al. 2004, 2006; Manthey et al. 2005; Mirzaei and Das 2007; Das and Mirzaei 2013). For example, Ataie-Ashtiani et al. (2002) have shown that, because of the presence of micro-heterogeneities, $\mathrm{P}^{\mathrm{c}}-\mathrm{S}_{\mathrm{w}}$ relationships measured in laboratory-scale domain may be sensitive to the imposed boundary conditions. The micro-heterogeneity effects on the $\mathrm{P}^{\mathrm{c}}-\mathrm{S}_{\mathrm{w}}$ relationship have been analysed in a number of other contexts too, e.g., effects of uniformly and randomly distributed heterogeneities, hydraulic parameters (e.g., entry pressure, intrinsic permeability), heterogeneity in particle size and intensity of the heterogeneities (e.g., Das et al. 2004, 2006; Ataie-Ashtiani et al. 2003). It is clear that the heterogeneity effects on the twophase flow properties are significant and they must be accounted for in the $\mathrm{P}^{\mathrm{c}}-\mathrm{S}_{\mathrm{w}}$ relationships.

Another important aspect of two-phase flow in porous media, which is directly linked to this study, is that the two-phase flow may not necessarily be at equilibrium $\left(\mathrm{dS}_{\mathrm{w}} / \mathrm{dt}=0\right)$, particularly at short periods after the start of the flow. Accordingly, the capillary pressure data obtained in an experiment may not be correct unless the $\mathrm{P}^{\mathrm{c}}-\mathrm{S}_{\mathrm{w}}$ relationships account for their dependence on the rate of change of fluid saturation $\left(\mathrm{dS}_{\mathrm{w}} / \mathrm{dt}\right)$. A number of experiments have shown that the $\mathrm{P}^{\mathrm{c}}-\mathrm{S}_{\mathrm{w}}$ curves at equilibrium and dynamic conditions are different (Topp et al. 1967; Smiles et al. 1971; Vachaud et al. 1972; Das and Mirzaei 2012, 2013; Mirzaei and Das 2013). The dependence of the capillary pressure curves on $\mathrm{dS}_{\mathrm{w}} / \mathrm{dt}$ is known as the dynamic effect in capillary pressure relationship (Hassanizadeh et al. 2002; Mirzaei and Das 2007; Das et al. 2007). Quantitatively, it is indicated by a coefficient called the dynamic coefficient $(\tau)$ which is calculated using Eq. (2): 


$$
\tau=-\frac{P_{d y n}^{c}-P_{s t a t}^{c}}{d S_{w} / d t}
$$

Here, $P_{d y n}^{c}$ is the dynamic capillary pressure defined as $P_{d y n}^{c}=P_{d y n}^{n w}-P_{d y n}^{w}$ and $P_{s t a t}^{c}$ is the capillary pressure at equilibrium conditions $\left(\mathrm{dS}_{\mathrm{w}} / \mathrm{dt}=0\right)$ defined as $P_{\text {stat }}^{c}=P_{\text {stat }}^{n w}-P_{\text {stat }}^{w}$. In the last decade, a number of studies have determined the significance of $\tau$ in different circumstances (Hassanizadeh et al. 2002; Dahle et al. 2005; Manthey et al. 2005; Das et al. 2007, 2014; Mirzaei and Das 2007, 2013; Peszynska and Yi 2008; Juanes 2009; Sakaki et al. 2010; Bottero et al. 2011; Mumford and O'Carroll 2011; Hanspal and Das 2012; Das and Mirzaei 2012, 2013; Hanspal et al. 2013; Hou et al. 2014; Zhang et al. 2014). In particular, it has been shown that the heterogeneity in porous media affects the dynamic coefficient significantly by altering the rate to flow equilibrium (Manthey et al. 2005; Mirzaei and Das 2007; Sakaki et al. 2010; Das and Mirzaei 2013). Das et al. (2007) and Das and Mirzaei (2013) show that the dynamic coefficient in heterogeneous domain is higher than in homogeneous domain implying that the two-phase flow system reaches equilibrium earlier in the homogeneous domain at a given water saturation.

The value of the dynamic coefficient is needed for not only understanding the dynamics of a two-phase flow in porous domain but also for correct application of the capillary pressure relationships. As such, the coefficient has been determined in the literature using both laboratory experimental work and computer simulations (at both pore and continuum scales). Recent studies have established that while the experiments for measuring the dynamic coefficient is a time consuming process lasting from many days to weeks (Das and Mirzaei 2013; Mirzaei and Das 2013), the mathematical and computer simulation tools based on flow physics are complex or computationally resource/time intensive (Manthey et al. 2005; Mirzaei and Das 2007; Hanspal and Das 2012). This issue becomes particularly important in the case of experiments involving heterogeneous domain where controlling the distribution and intensity of heterogeneity (defined as the ratio of the volume of heterogeneity to the total sample volume). Relating these parameters to the dynamic coefficient is not straightforward and, therefore, simple heterogeneity patterns (e.g., layers) are often created for this purpose (Sakaki et al. 2010; Das and Mirzaei 2013; Mirzaei and Das 2013). On the other hand, the mathematical tools for calculating the dynamic coefficient for heterogeneous domain rely on incorporating spatial distributions of the heterogeneity patterns (Manthey et al. 2005; Mirzaei and Das 2007; Sakaki et al. 2010). Statistical tools, such as the Markov chain method, may be applied to create 'artificial' heterogeneity within porous media and carry out simulations for these media to determine the dependence of the dynamic coefficient on the domain heterogeneity. The 'artificial' heterogeneous domain in this case does not necessarily need to represent a real domain, but it provides a good way to relate the dynamic coefficient to realistic heterogeneities. To the authors knowledge, such an approach has not been used for calculating the dynamic coefficient.

Irrespective of how the heterogeneity patterns in porous media are accounted for in the calculation of the dynamic coefficient, it is safe to assume that the exact distribution and intensity of the heterogeneity in the domain are not always the most critical parameters. These parameters are often unknown or cannot be directly determined. It is the effective/average twophase flow behavior (e.g., $\mathrm{P}^{\mathrm{c}}-\mathrm{S}_{\mathrm{w}}$ curves) that is of the most interest in general. This raises an important question, namely, is it always necessary to run lengthy, complicated and costly laboratory experiments to determine the dynamic coefficient in heterogeneous domain given that we are mostly interested in average two-phase behavior? An alternate approach for the determination of the dynamic coefficient, which would be easier in comparison to the costly numerical simulation tools, without relying on expert knowledge of the two-phase porous 
media flow theory and complex procedures for averaging flow behaviour in porous domains, would be very useful. This alternate approach should be computationally less demanding, alleviating any detailed sub-sample scale level domain information requirements. In this regard, a generic model, such as the one presented in this paper, which is focused on doing a specific task while addressing these issues, is of great need.

In order to address the above mentioned issues, this paper presents a novel artificial neural network (ANN) architecture that has been developed to predict dynamic coefficients in heterogeneous porous domains.

An ANN consists of a group of interconnected processors known as neurons (Deka and Quddus 2014; Demuth et al. 2008; Graupe 2007) which mimic aspects of a human brain by learning, recognizing problem patterns and data identification/classification, and exploring complex non-linear mathematical relationships between the input and output responses of a system. Incorporation of multiple neurons or processors in different layers (input, output and hidden) of the ANN architecture (feed-forward or feed-backward) can determine the performance of the ANN in terms of efficiency and computational run-times (Hanspal et al. 2013; Ren 2009; Kurtulus and Razack 2006). In the literature, many such examples of ANN based tools in the context of flow and transport in porous media can be referred, e.g., determination of dynamic effect in homogeneous porous media (Hanspal et al. 2013), oil well logging and testing (Baldwin 1991; Kumoluyi and Daltaban 1994; Elkamel et al. 1996), prediction of petrophysical properties (Karimpouli et al. 2010; Omole et al. 2009; Boadu 2001), simulation of groundwater contamination, pollutant infiltration and surface run-off (Akratos et al. 2008; Tabach et al. 2007; Parida et al. 2006), and immiscible flooding simulations (Elkamel et al. 1996; Gharbi et al. 1995; Li and Lake 1995; Shook et al. 1992). However, an ANN-based tool that can specifically characterize the dynamic coefficient in heterogeneous porous domains, addressing the points discussed above, is still missing. Therefore, in this work, we present an ANN approach which incorporates the intensity of heterogeneity and average porous media permeability as input parameters, in addition to other important parameters of the two-phase flow system to quantify the dynamic coefficient. As there is a reasonably good understanding on how the dynamic coefficient depends on the porous domain heterogeneity (e.g., Manthey et al. 2005; Mirzaei and Das 2007; Das and Mirzaei 2013), we believe that such an approach is possible. However, it must be recognized that the amount of quantitative data are still limited. As discussed earlier, ANN is a very good modelling option in the context of this work as it can be used to determine the dynamic coefficient in heterogeneous domains much beyond the span of typical laboratory scale experiments and numerical modelling schemes which are often very tedious. Importantly, ANN enables a continuous learning process in which the input variables can be continuously updated creating a larger database for better training and validation of the ANNs, hence, enabling better predictions in the future. Therefore, as more data become available in the future relating the dynamic coefficient to the heterogeneity, the ANN can be improved further. In this paper, six key parameters namely, water saturation, intensity of heterogeneity, average permeability, fluid density ratio, fluid viscosity ratio and temperature are used to train and develop the neural network in order to determine the best performing model through variation of neuron numbers. The temperature and the fluid properties are kept constant, so as to determine the significance of micro-heterogeneities on the dynamic coefficient more accurately.

Conceptually, the presented approach is somewhat motivated by an ANN structure previously reported to compute dynamic coefficient in homogeneous domain (Hanspal et al. 2013). However, the work in this paper does not have any other link to the previous work as the objective in this paper is completely different and we use different ANN structure and reference data sets. In the present work, we include an additional independent variable, i.e., 
intensity of heterogeneity (Das et al. 2004; Mirzaei and Das 2007) to account for the amount of heterogeneity in the computational domain. Furthermore, an average permeability of the heterogeneous domain is used instead of the homogeneous domain permeability as previously used by Hanspal et al. (2013). Similar to Hanspal et al. (2013), we argue that the effects of any other system parameters (e.g., interfacial tension, entry pressure of porous domain) that are not explicitly accounted for in the ANN structure are lumped in the values of the saturation (input) and dynamic coefficient (output). Of course, an ANN such as the present one may include as many parameters as one would like as input and output variables. However, the results in this paper seem to suggest that an ANN structure with 6 input and 1 output variables can incorporate the salient features of the dependence of dynamic coefficient on the intensity of micro-heterogeneity in porous media.

\section{Modelling Approach}

The ANN based tool for calculating the dynamic coefficient is implemented using the ANN toolbox in MATLAB. The developed ANN model uses different input/output relationships from those previously used by Hanspal et al. (2013). The ANN modeling approach involves the implementation, testing and validation of the ANN structure coupled with the use of an average permeability calculation for heterogeneous domains. Hanspal et al. (2013) showed that it is possible to obtain reasonable estimates of the values of dynamic coefficient (output variable) with 5 input parameters for homogeneous porous domain. In the present context, intensity of heterogeneity is used as an additional input parameter to define the ratio of the volume of heterogeneity to total volume of the sample (Das et al. 2004; Mirzaei and Das 2007).

Due to insufficient availability of the dynamic coefficient data for ANN training and validation a function 'cftool' in MATLAB (MATLAB 8, the MathWorks Inc., Cambridge, UK, 2012) is utilized to generate additional data on the dynamic coefficient as a function of different intensities of heterogeneity, by fitting curves against the simulation data listed by Mirzaei and Das (2007). The cftool function allows one to fit the data with no pre-assumption on the form of the fitting parameters and provide a code that can generate additional data. Using this approach, we have generated additional artificial data which have been used as the reference data for developing the ANNs. The values of intensity of heterogeneity used in this paper are the same as previously used by Mirzaei and Das (2007). The extrapolated data sets consisted of 110 data points, which were utilized for ANN development, testing and validation. We realize that this may be viewed as a limited dataset for developing ANN. However, as more experimental and modelling data on the relationship between the dynamic coefficient and intensity of heterogeneity become available in the future, they can be incorporated in the developed ANN.

The important statistics of the data used in this work are provided in Table 1. The fluid property ratios listed in Table 1 can be temperature-dependent. The temperature dependency of these variables can be determined using correlations reported by Hanspal and Das (2012) and implemented in the ANN structure. However, for the purpose of this paper, the temperature and the fluid properties are kept constant so as to determine the significance of microheterogeneities on the dynamic coefficient.

In this work, a back propagation algorithm in feed-forward neural networks has been used which is viewed as a very powerful ANN learning algorithm (Orchard and Phillips 1991). It has been previously shown that a feed-forward ANN architecture (Rumelhart et al. 1986) with back propagation (Werbos 1995) offers a robust solution for complex non-linear problems provided that there are sufficient neurons in the hidden layer(s) (van der Heyden 1996) and the 


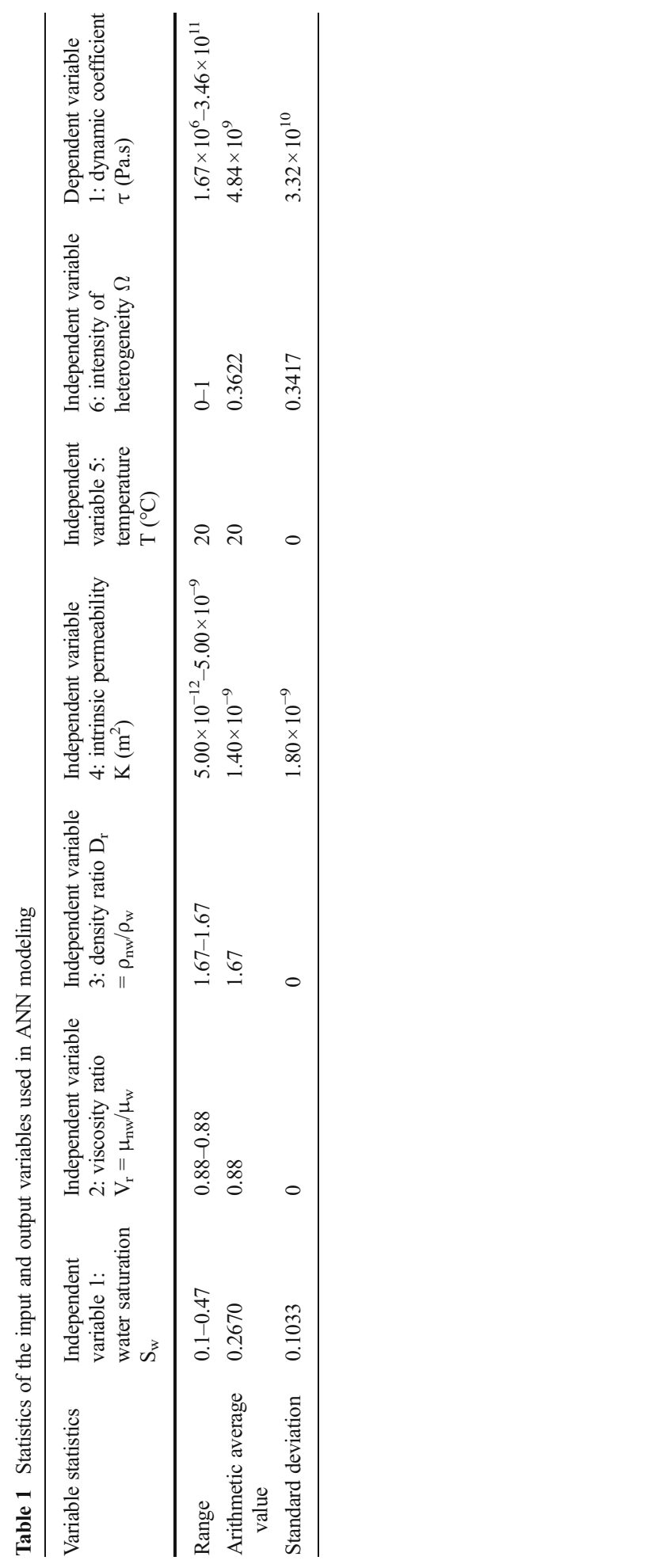


training data set is large enough for the learning process to avoid convergence at a local minimum (Haykin 1999). Henceforth, in the present work we use MATLAB's ANN toolbox to implement a multilayer feed-forward network based on a back-propagation training algorithm to describe the non-linear relationships between the dynamic coefficient and physical properties characterizing the heterogeneity in multiphase porous transport. LevenbergMarquardt algorithm (Levenberg 1944) is generally used to determine the robustness and time-efficiency of a feed-forward neural network which was implemented in this work too. In conjunction with the standard pre-and post-processing procedures, the back-propagation algorithm was implemented in four sequential steps, namely: (a) data assimilation; (b) network object creation; (c) network training; and (d) network response simulation. Particular transfer function, namely, 'log-sig', 'tan-sig' and 'purelin' were utilized to yield positive and physically meaningful dynamic coefficients at all water saturation inputs during the network training process. The reference data (RD) were split by three data-sets: 1) training set: $60 \%$ of $\mathrm{RD} ; 2$ ) validation set: $20 \%$ of RD; and 3) testing set: $20 \%$ of RD. The training data set was scaled up to normalize its mean and standard deviation to values of zero and unity, respectively, as done previously by Hanspal et al. (2013). No other scaling functions for the inputs and outputs were used. The segregated data-sets were then utilized for: (a) training (i.e., to evaluate and recognize behavioural patterns in data); (b) validation (i.e., to assess network generalization characteristics); and (c) testing (i.e., to provide an independent evaluation of network generalization for new data that the network did not experience before).

Network training was carried out until there was a continuous decrease of the network's errors on the validation vectors. Training was conducted multiple times using five-fold crossvalidation to ensure that each of the 110 data points was a part of the training-set. To prevent data over-fit by the ANN, a relatively large data-set was used in comparison to the number of data points required for plotting the $\tau-S_{\mathrm{w}}$ curve. Stratified sampling was applied to ensure that the statistics of the testing and training data matched closely. In addition, different performance indicators, namely the mean square error (MSE), sum squared error (SSE), average absolute relative error (AARE), Nash-Sutcliffe efficiency coefficient (E), and Pearson product moment coefficient of correlation (R) (Hanspal et al. 2013; Jain et al. 2001) were utilized to assess the robustness and accuracy of the ANN models with different neuron numbers. Performance parameters were computed for the entire dataset (i.e. both input and output data).

A calculation technique outlined by Stam and Ziji (1992) was utilized to compute the average permeability of heterogeneous domain. This value was then used as an input parameter in the ANN. Heterogeneous domains consisting of coarse and fine sands, as used by Mirzaei and Das (2007), were chosen for providing the reference data for the dependence of the dynamic coefficient on the intensity of heterogeneity. This is to ensure that the data for the dynamic coefficient and the heterogeneous pattern correspond to each other. Furthermore, the permeability of individual heterogeneous pattern in a domain, as well as the exact distribution of the pattern, are provided by Mirzaei and Das (2007), which can be used to compute average permeability using the method suggested by Stam and Ziji (1992).

In heterogeneous porous media, the average permeability can be computed by utilizing permeability at several points within the computational domain. The average permeability based on small scale units was calculated by Stam and Ziji (1992) as in Eq. (3)

$$
\langle\langle k\rangle\rangle=2 a\left\{\int_{-a}^{+a}[k(x)]^{-1} d x\right\}^{-1}
$$

Here, $\langle\langle k\rangle\rangle$ is the average permeability $\left[\mathrm{m}^{2}\right], 2 \mathrm{a}$ is the horizontal dimension of grid-blockscale $[\mathrm{m}]$, and $\mathrm{k}(\mathrm{x})$ is the permeability of a smaller block scale grid within heterogeneous 
porous domain. Warren and Price (1961) and Jensen (1991) suggested that the average permeability should be calculated at sub-block grid scale for uncorrelated permeability distribution. Motivated by these, the average permeability is computed using Eq. (4) under the assumption of uncorrelated permeability distribution

$$
K_{a v g}=\exp \left[\frac{\sum_{i=1}^{N}\left(2 a \cdot \ln \left(k_{i}\right)\right)}{\sum_{i=1}^{N} 2 a}\right]
$$

Here, $\mathrm{K}_{\mathrm{avg}}$ is the average permeability $\left[\mathrm{m}^{2}\right], 2 \mathrm{a}$ is the horizontal dimension of the sample [m], and $\mathrm{N}$ is the total number of samples [dimensionless]. In this case, a heterogeneous porous media, consisting of various coarse and fine sand grid-block units are used as illustrated in Fig. 1 and Table 2.

The permeability of heterogeneous porous sample lies between the permeability of pure coarse and fine sand samples, which are $5 \times 10^{-9}$ and $5 \times 10^{-12} \mathrm{~m}^{2}$, respectively (Mirzaei and Das 2007). The permeability values used in this work, corresponding to different intensity values (Mirzaei and Das 2007), are illustrated in Table 2.

As mentioned earlier, the function cftool in MATLAB was employed to estimate the best curve-fits (see Fig. 2) describing the average permeability as a function of intensity of heterogeneity. Similarly, curve-fits were determined to describe the dynamic coefficient as a function of water saturation at different heterogeneity intensity values of $0,0.207,0.331,0.372$ and 1, respectively, as shown in Fig. 3.

\section{Results and Discussion}

Results obtained from the developed ANN model during various stages of network training, performance testing and validation were used to assess the predicting capabilities and evaluate most significant variables affecting the output. These results are discussed in the following sections.

\subsection{ANN Training and Performance}

The post regression plots presented in Fig. 4 compare the training and the predicted data. In principle, the best linear fit is described by a slope $(\mathrm{M})=1$, y-intercept $(\mathrm{B})=0$ and a mean square error $(\mathrm{MSE})=1$. The $\mathrm{M}, \mathrm{B}$ and MSE values obtained for different ANN models with varying neuron numbers are listed in Table 3.

It was found that the best performance in terms of slope is the network with 3 neurons, whilst in terms of y-intercept it is the network with 12 neurons. Post regression analysis plots for various neuron numbers in different single hidden layer are shown in Fig. 4. By comparing the mean square error (MSE) for different neuron numbers, as illustrated in Fig. 4 and Table 3, it is inferred that 15 neurons in a single hidden layer provide the best performance in terms of MSE closest to unity. It is believed that the network predicting the highest MSE has the best performance. In addition to MSE, other performance indicators, such as the sum square error (SSE), the average absolute relative error (AARE), the Nash-Sutcliffe efficiency coefficient (E) and the Pearson product moment coefficient of correlation $(\mathrm{R})$, were determined to assess the model performances. Computed values for these performance indicators are listed in Table 4. It 


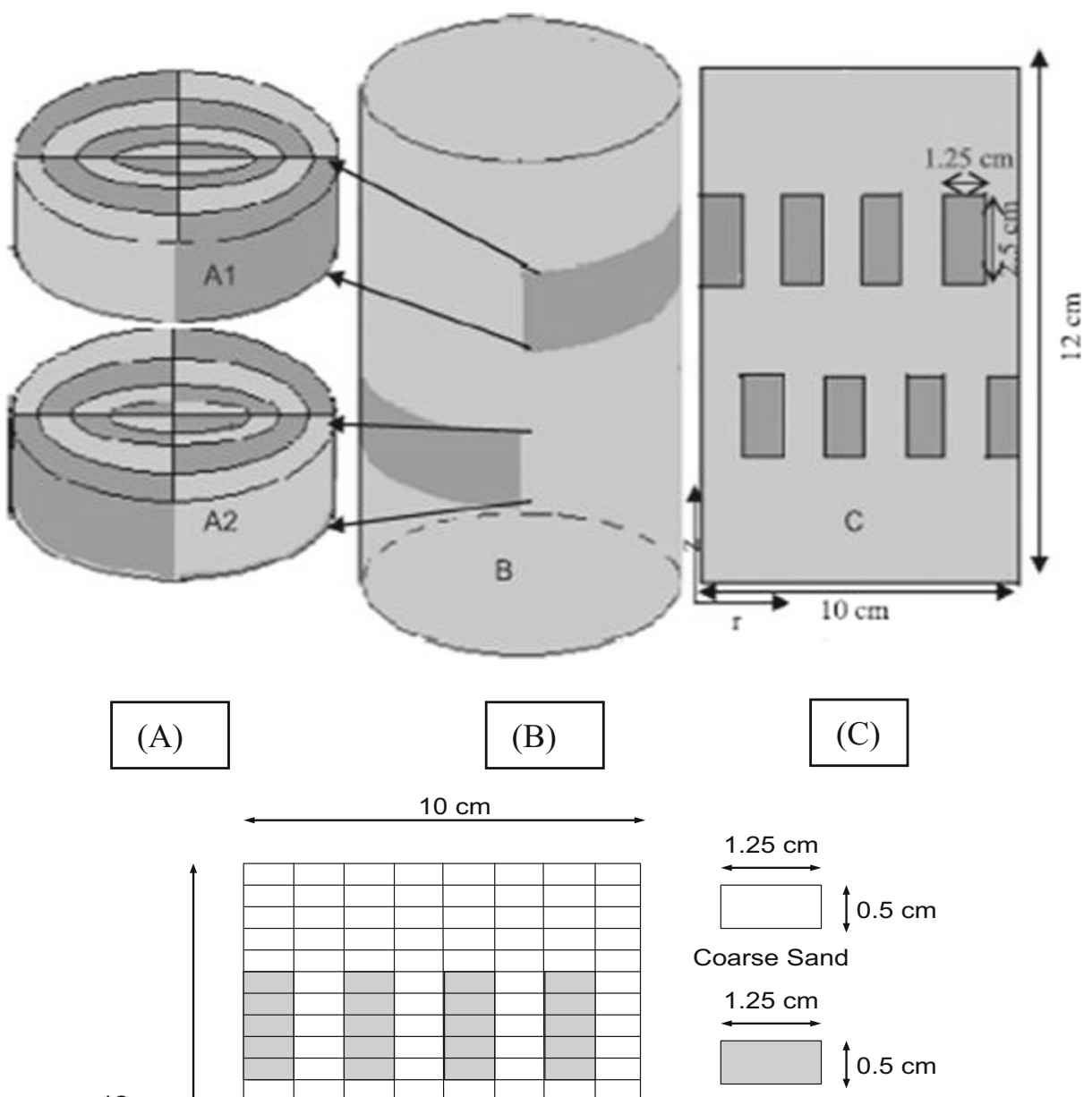

$12 \mathrm{~cm}$

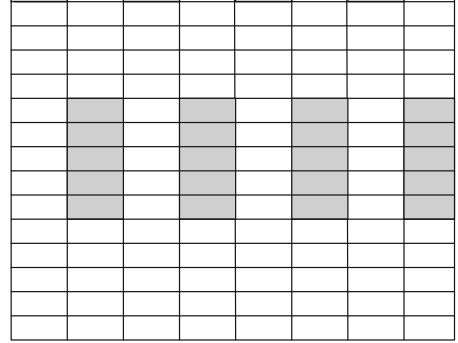

Fine Sand

(D)

Fig. 1 Intensity $(\omega)$ and distributions of micro-heterogeneities $(\omega=0.207)$ in the form of small fine sand blocks (dark grey blocks) embedded in coarse sand background $(A 1, A 2)$ a $\mathrm{R}-\Theta$ view of heterogeneity distribution $\mathbf{b} 3 \mathrm{D}$ heterogeneous cylindrical domains $\mathbf{c} 2 \mathrm{D}$ rectangular $(\mathrm{R}-\mathrm{Z})$ section of heterogeneity distribution in cylindrical domains (Mirzaei and Das 2007) d sub-grid block units of computational domain used for estimating average permeability (please see Table 2) 
Table 2 Computed average permeability at different values of intensity of heterogeneity

\begin{tabular}{lllr}
\hline $\begin{array}{l}\text { Intensity of heterogeneity as used by } \\
\text { Mirzaei and Das (2007) }\end{array}$ & $\begin{array}{l}\text { Homogeneous fine sand } \\
\text { No of blocks for } \\
\text { permeability calculation }\end{array}$ & $\begin{array}{l}\text { Homogeneous coarse sand } \\
\text { Number of blocks for } \\
\text { permeability calculation }\end{array}$ & $\begin{array}{l}\text { Average } \\
\text { permeability } \\
\left(\mathrm{m}^{2}\right)\end{array}$ \\
\hline 0 & 0 & 192 & $5 \times 10^{-9}$ \\
0.371 & 72 & 120 & $3.749 \times 10^{-10}$ \\
0.331 & 64 & 128 & $5 \times 10^{-10}$ \\
0.207 & 40 & 152 & $1.186 \times 10^{-9}$ \\
1 & 192 & 0 & $5 \times 10^{-12}$ \\
\hline
\end{tabular}

is found that for all performance indicators, the models with 12 and 15 neurons display the best results, while those with 3 neurons perform the worst.

\subsection{Validation of ANN}

As illustrated in the previous section, ANN models with 12 and 15 neurons perform the best. Henceforth, the reference data for validation have been compared against the 12 and 15 neuron ANN models. Fig. 5 shows that the 15 neurons model compares better to the reference data than the 12 neurons model. It is, then, concluded that the 15 neurons ANN model (Fig. 5) is the most appropriate for quantifying the dynamic coefficients associated with flows in heterogeneous porous media.

\subsection{Prediction of Dynamic Coefficient Using ANN}

After the ANN model training and performance assessments were completed, the 15 neurons structure was utilized to determine the dynamic coefficient values for new input data-sets corresponding to intensity values ranging between 0.4 and 0.9. Fig. 6 illustrates that the dynamic coefficients decrease with an increase in water saturation. Furthermore, with an increase in the intensity of heterogeneity levels at any given water saturation, the dynamic coefficient increases. This finding is in agreement with the results presented by Manthey et al. (2005), Mirzaei and Das (2007) and Das and Mirzaei (2013).

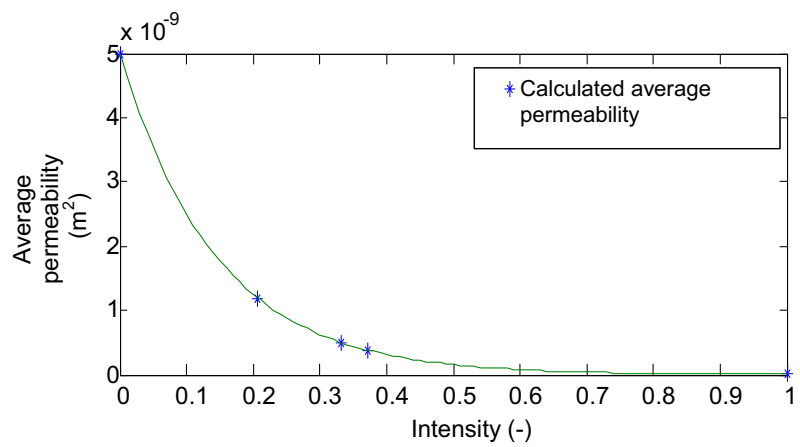

Fig. 2 Non-linear fit for average permeability as a function of intensity of heterogeneity 


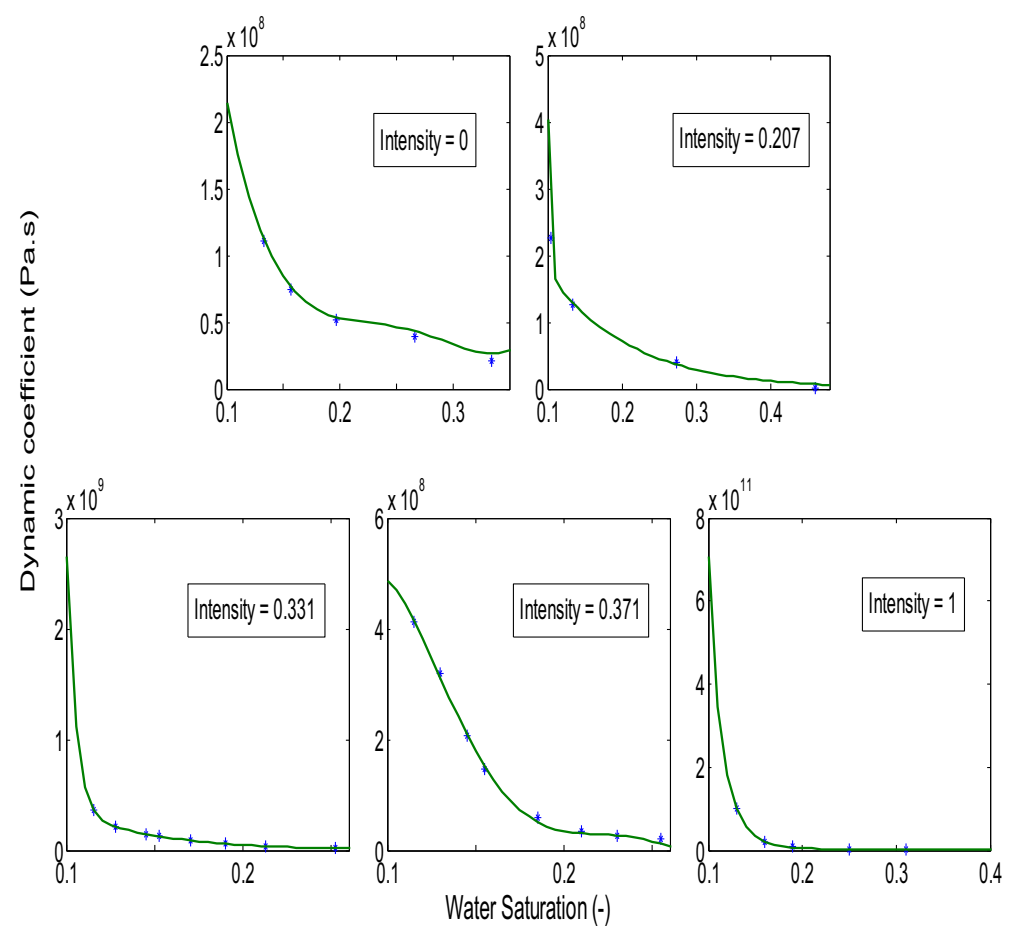

Fig. 3 Non-linear fits for dynamic coefficient $(\tau)$ as a function of water saturation for different intensity of heterogeneity. In this paper, the heterogeneous domain contains fine sand embedded in coarse sand and the intensity of heterogeneity is defined as the ratio of the volume of fine sand to the total volume of the sample. The reference data used in this paper were taken from Mirzaei and Das (2007)

\subsection{Determination of the Most Significant Variable Affecting the Dynamic Coefficient}

As shown in a number of publications including this paper, the dynamic coefficient is affected by a number of variables. This, however, raises the question, e.g., which of these variables are the most significant in determining the value of the coefficient. This issue can be addressed in the context of implementing an ANN using MATLAB. It is assumed that under the effects of high weight attribution, the output (i.e., the dynamic coefficient) from the MATLAB will be highly affected. Hence, for the best performing ANN model with 15 neurons, the effects of weight attribution were incorporated. The neural network structure in this paper consists of a single layer which requires two weight coefficients and two bias values consisting of input and hidden layers in the form of input weight, layer weight, bias 1 and bias 2 . Three parameters, namely density ratio, viscosity ratio and temperature, have been fixed for the six parameters considered in the ANN model. Weight coefficients are, therefore, only associated with varying other parameters which are water saturation, intensity of heterogeneity and average permeability. Due to the existence of 15 neurons in the network, there are 15 sets of weight coefficients associated with each of these parameters. Table 5 shows the weight coefficients in the ANN model. It is possible that higher absolute average weight coefficients might affect the solution strongly. It was, however, decided to use the absolute average instead of the normal average. Negative weight coefficients indicate how strong the impact will be on an inverse relationship. Moreover, layer weight, bias 1 and bias 2 can be ignored since the 
3 Neurons

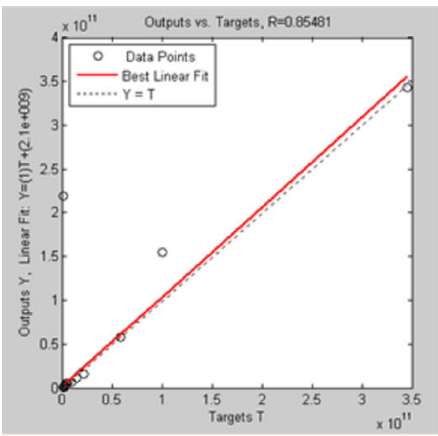

8 neurons
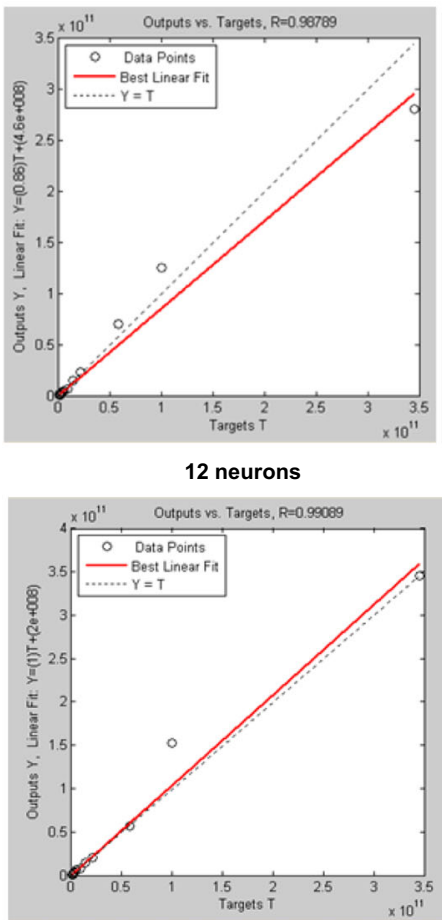

12 neurons

5 Neurons

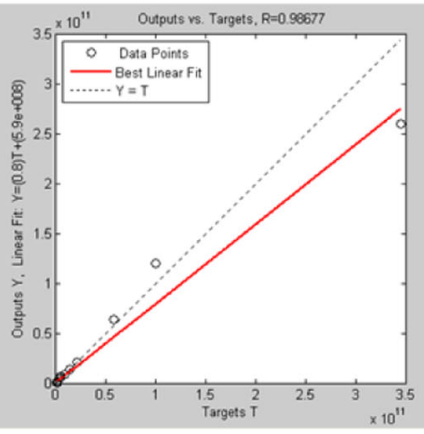

10 neurons

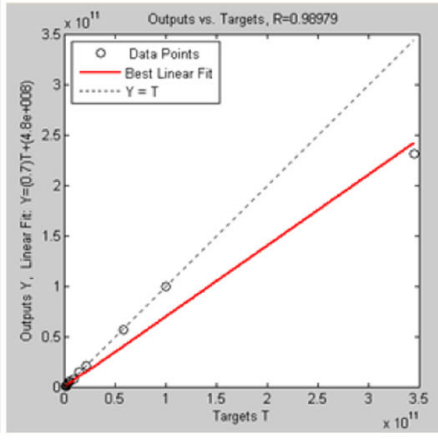

15 neurons

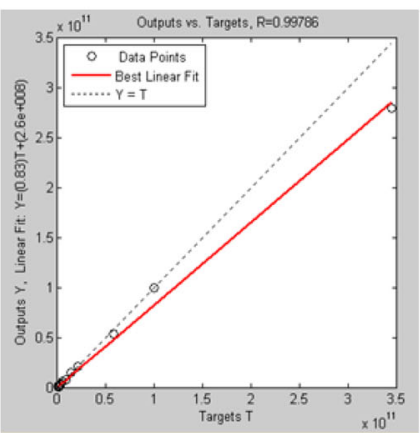

18 neurons

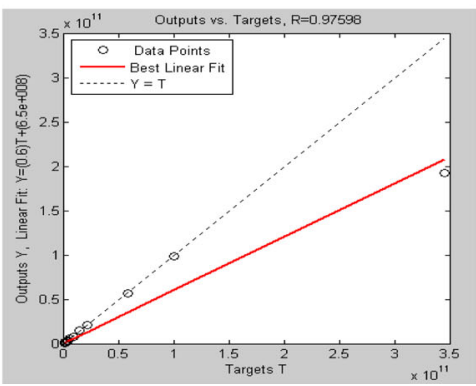

Fig. 4 Post regression analysis in different single hidden layer for different neuron numbers 
Table 3 Comparison of y-intercept (B), slope (M) and mean square error (MSE) for regression analysis

\begin{tabular}{|c|c|c|c|c|c|c|c|}
\hline Neurons & 3 & 5 & 8 & 10 & 12 & 15 & 18 \\
\hline M & 1.0253 & 0.796 & 0.8552 & 0.7024 & 1.0379 & 0.8277 & 0.6018 \\
\hline B & $2.10 \mathrm{E}+09$ & $5.88 \mathrm{E}+08$ & $4.63 \mathrm{E}+08$ & $4.80 \mathrm{E}+08$ & $2.04 \mathrm{E}+08$ & $2.56 \mathrm{E}+08$ & $6.46 \mathrm{E}+08$ \\
\hline Mean Square Error (MSE) & & & & & & & \\
MSE $=\frac{1}{N} \sum_{i=1}^{N}(t(i)-a(i))^{2}$ & & & & & & & \\
\end{tabular}

Please note that the color-filled cells correspond to the best values of M, B and MSE on the bases that the best values should be the closest to $M=1, B=0$ and $M S E=1$

$N$ total number of data points predicted

$t$ target value of dynamic coefficient $(\tau)$

$a$ network output of dynamic coefficient $(\tau)$

addition or multiplication to the neuron will affect all the concerned parameters by the same amount.

In Table 5, it is observed that the percentage of absolute average is the largest for the intensity of heterogeneity at $38.76 \%$. Next, it corresponds to water saturation at $37.6 \%$ and permeability at $23.64 \%$. This suggests that there is not a significant difference between the water saturation and heterogeneity effects on the dynamic capillary pressure effects. It also implies that the influence of both parameters on the dynamic coefficient is similar and it is much greater in comparison to the effects of average permeability. Therefore, it is concluded that variations of water saturation and intensity of heterogeneity are more important than the average permeability effect concerning the values of dynamic coefficient.

\subsection{Computational Run-Times}

The maximum run-time required for the ANN model training and validation based on the existing data set was less than $2 \mathrm{~h}$. Furthermore, once the model was completely trained, the computational run-time associated with new predictions of dynamic coefficient values was in the order of minutes. This represents almost a 100-125 fold decrease in comparison to the

Table 4 Overall error performance comparison using different performance indicators

\begin{tabular}{|c|c|c|c|c|c|c|c|}
\hline \multirow{2}{*}{$\begin{array}{l}\text { Error performance indicators and the } \\
\text { equations to calculate them }\end{array}$} & \multicolumn{7}{|c|}{ Number of Neurons } \\
\hline & 3 & 5 & 8 & 10 & 12 & 15 & 18 \\
\hline Sum squared error (SSE) & & & & & & & \\
\hline $\mathrm{SSE}=\sum_{i=1}^{N}\left(S_{\mathrm{obs}}-S_{\mathrm{cal}}\right)^{2}$ & $5.13 E+22$ & $7.69 \mathrm{E}+21$ & $5.10 \mathrm{E}+21$ & $1.30 \mathrm{E}+22$ & $2.81 \mathrm{E}+21$ & $4.30 \mathrm{E}+21$ & $2.33 E+22$ \\
\hline $\begin{array}{c}\text { Average absolute relative error } \\
\text { (AARE) }\end{array}$ & & & & & & & \\
\hline $\mathrm{AARE}=\frac{1}{N} \sum_{i=1}^{N}\left|\frac{S_{\mathrm{cal}}-S_{\mathrm{obs}}}{S_{\mathrm{obs}}}\right| \times 100$ & $1.22 \mathrm{E}+03$ & $1.47 \mathrm{E}+02$ & $9.59 E+01$ & $6.80 \mathrm{E}+01$ & $7.86 \mathrm{E}+01$ & $5.02 E+01$ & $5.64 \mathrm{E}+01$ \\
\hline $\begin{array}{l}\text { Nash-Sutcliffe efficiency coefficient (E) } \\
\qquad E=1-\frac{\sum\left(S_{\mathrm{cal}}-S_{\mathrm{obs}}\right)^{2}}{\sum\left(S_{\mathrm{obs}}-\bar{S}_{\mathrm{obs}}\right)^{2}}\end{array}$ & $6.07 E-01$ & $9.41 \mathrm{E}-01$ & $9.61 \mathrm{E}-01$ & $9.00 \mathrm{E}-01$ & $9.79 \mathrm{E}-01$ & $9.67 \mathrm{E}-01$ & 8.22E-01 \\
\hline $\begin{array}{l}\text { Pearson product moment coefficient of } \\
\text { correlation (R) } \\
R=\frac{\sum\left(S_{\mathrm{obs}}-\bar{S}_{\mathrm{obs}}\right) \times\left(S_{\mathrm{cal}}-\bar{S}_{\mathrm{cal}}\right)}{\sqrt{\sum\left(S_{\mathrm{obs}}-S_{\mathrm{obs}}\right)^{2} \sum\left(S_{\mathrm{cal}}-\bar{S}_{\mathrm{cal}}\right)}}\end{array}$ & 8.55E-01 & 9.87E-01 & $9.88 \mathrm{E}-01$ & $9.90 \mathrm{E}-01$ & $9.91 \mathrm{E}-01$ & $9.98 \mathrm{E}-01$ & $9.76 \mathrm{E}-01$ \\
\hline
\end{tabular}

Please note that the color-filled cells correspond to the best values of SSE, AARE, E and R

$\mathrm{N}=$ total number of data points predicted

$S_{\text {obs }}=$ observed value of dynamic coefficient $(\tau) ; \bar{S}_{o b s}=$ average observed dynamic coefficient $(\tau)$

$S_{c a l}=$ calculated value of dynamic coefficient $(\tau) ; \bar{S}_{c a l}=$ average calculated dynamic coefficient $(\tau)$ 


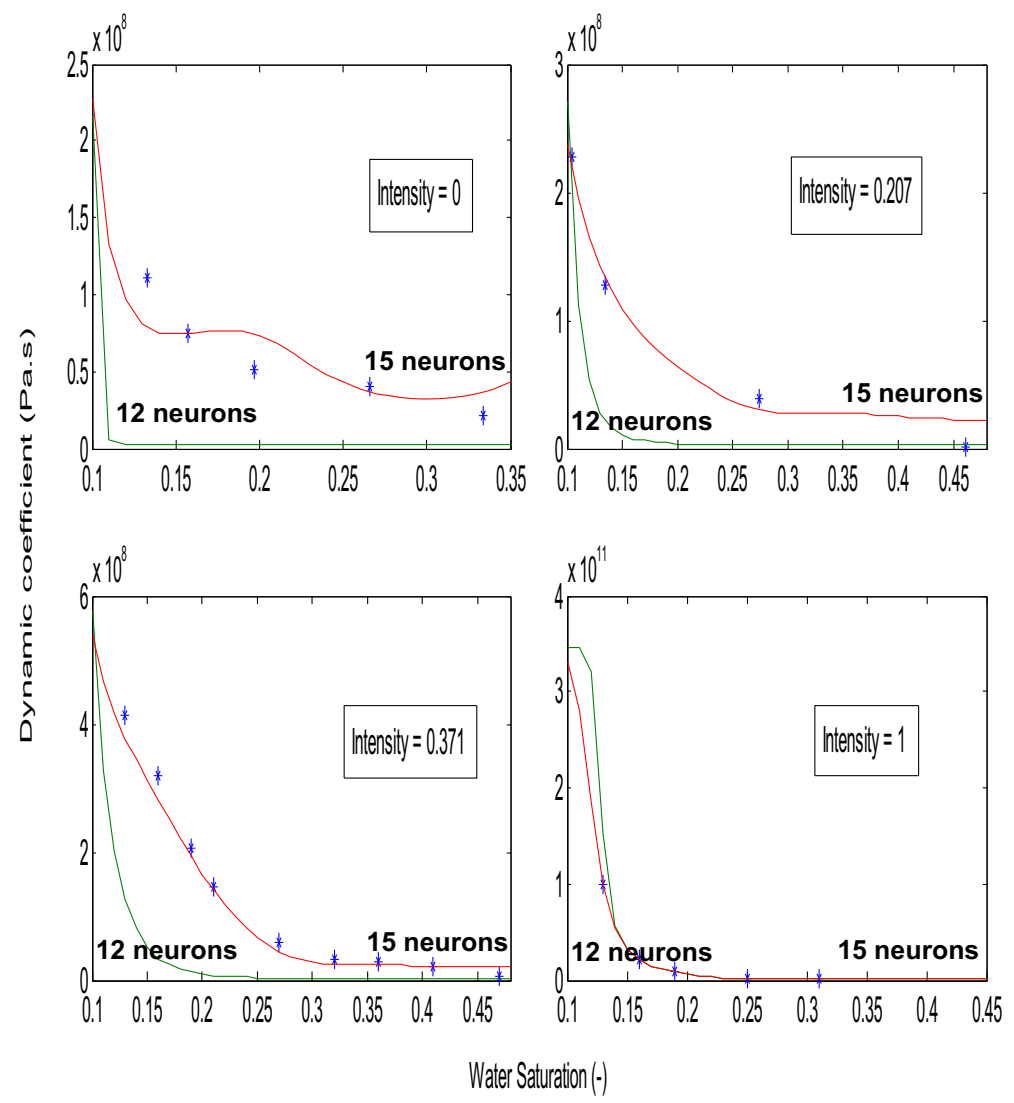

Fig. 5 Comparison of reference dynamic coefficient values (shown by star symbols) from Mirzaei and Das (2007) and two best performing ANN models, i.e., 12 neurons (green lines) and 15 neuron (red lines) for different intensities of heterogeneity

computational time previously required for flow physics-based simulations such as the STOMP simulator (Hanspal et al. 2013). Numerical simulations are both resource and time intensive, with run times for the determination of the dynamic coefficient ranging from several

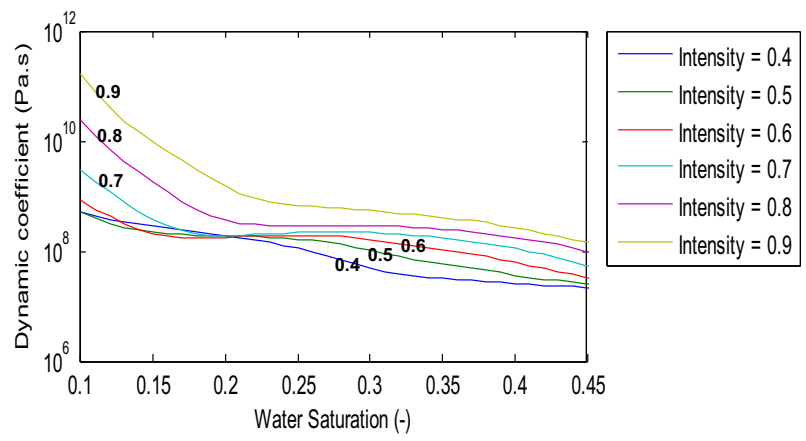

Fig. 6 Prediction of dynamic coefficient as a function of water saturation for various hypothetical intensities of heterogenity 
Table 5 Weight coefficients in the network with 15 neurons

\begin{tabular}{|c|c|c|c|c|c|c|}
\hline \multirow{2}{*}{$\begin{array}{l}\text { Number of } \\
\text { neurons }\end{array}$} & \multicolumn{3}{|c|}{ Weight $\{1,1\}$} & \multirow[t]{2}{*}{ Bias 1} & \multirow{2}{*}{$\begin{array}{l}\text { Weight } \\
\{2,1\}\end{array}$} & \multirow[t]{2}{*}{ Bias 2} \\
\hline & $\begin{array}{l}\text { Water } \\
\text { saturation }\end{array}$ & $\begin{array}{l}\text { Intensity of } \\
\text { heterogeneity }\end{array}$ & $\begin{array}{l}\text { Average } \\
\text { permeability }\end{array}$ & & & \\
\hline 1 & 2.9526 & 5.4308 & 0.9037 & -7.6671 & -0.6219 & 1.6401 \\
\hline 2 & -0.1118 & -6.6249 & 1.5266 & -6.1456 & -0.1676 & \\
\hline 3 & -5.1815 & 5.2409 & 4.3293 & 4.0475 & -0.886 & \\
\hline 4 & 1.688 & -4.5288 & -4.5282 & -4.4163 & -0.6926 & \\
\hline 5 & -6.8397 & 1.8915 & 2.0673 & 3.9705 & -0.4091 & \\
\hline 6 & 2.6225 & -6.0828 & -0.8715 & -2.4346 & 0.5524 & \\
\hline 7 & 3.3359 & 2.0432 & -5.8187 & -1.8891 & -1.3572 & \\
\hline 8 & -3.7859 & -5.3024 & 4.6061 & -0.8674 & -0.3847 & \\
\hline 9 & -5.8836 & 4.0875 & 1.9875 & -3.0566 & -2.1748 & \\
\hline 10 & -6.8626 & 3.9373 & 1.5429 & -4.8906 & 2.1642 & \\
\hline 11 & -8.4861 & 5.7804 & 1.5657 & 2.0452 & 0.5502 & \\
\hline 12 & -8.2795 & 4.802 & 3.8707 & -9.7142 & 7.8014 & \\
\hline 13 & 4.0244 & 3.008 & -1.3649 & 7.1783 & -0.9606 & \\
\hline 14 & 3.4999 & -2.9192 & 6.2869 & 4.7972 & -2.0012 & \\
\hline 15 & 2.9939 & -6.9094 & 0.5734 & 6.8966 & -2.1583 & \\
\hline Average & 4.436527 & 4.572607 & 2.78956 & & & \\
\hline Percentage & 37.60185 & 38.7552 & 23.64296 & & & \\
\hline
\end{tabular}

IW $\{1,1\}$ is input weight matrix, $\operatorname{LW}\{2,1\}$ is layer weight matrix, b1 is bias1, b2 is bias2

days to weeks. Furthermore, there are additional time periods required for the post-processing of the simulated data. The developed ANN, therefore, represents a cost effective and robust approach to simulate new dynamic coefficient values in unknown regimes of heterogeneity, as illustrated in this study, provided that there exists a minimal amount of information in the form of reliable data sets either extracted from experiments or from computational flow physics simulations.

\section{Conclusions}

In this work, the application of artificial neural network (ANN) technique is illustrated to successfully compute a parameter of dynamic two-phase flow in porous media, namely, the dynamic capillary pressure coefficient, by considering micro-heterogeneity in porous media as a key parameter. In general, ANNs are capable of producing reliable results when the knowledge base is large and they cover all possible combinations in the range of values of input-output parameters. In this study, as discussed, the database was small. However, it was shown that the developed ANNs were capable of providing physically realistic values of the dynamic capillary pressure coefficient. This provides the confidence that the developed ANN can perform accurately. Block-grid computations were conducted through inclusion of nonuniform permeability effects within the computational domain. Curve-fitting techniques were utilized to provide enough data through computation of dynamic coefficient values at several intensity values. Post-regression analysis revealed that an ANN model consisting of a single layer with 3 neurons was the worst performer, yielding a mean square error equal to 0.85481 . 
On the other hand, a 15-neurons model was the best performer. It provided excellent statistics for mean square error (MSE), sum square error (SSE), average absolute relative error (AARE), Nash-Sutcliffe efficiency coefficient (E), and Pearson product moment coefficient (R) equal to $0.9979,4.30 \times 10^{21}, 50.2,0.967$ and 0.998 , respectively. Although the 12-neurons ANN showed the best performance in terms of SSE and E, which were $2.81 \times 10^{21}$ and 0.979 , respectively, the 15 -neurons ANN provided dynamic coefficient predictions much closer to the data of Mirzaei and Das (2007). In terms of the overall predictions, an ANN architecture with 15 neurons yielded practical results in the range of water saturation between 0.1 and 0.45 . Furthermore, all the ANN simulations predicted that the dynamic coefficient increases with an increase in intensity of heterogeneity and a decrease in water saturation, findings consistent with other studies. It is shown that the developed ANN model was able to predict dynamic coefficients under unknown conditions in relatively small run-times, signifying a speed-up by a factor of 125 in comparison to real experimental studies or full-scale computational flow physics- based simulations.

Acknowledgment The work in this paper was conducted in the framework of Engineering and Physical Research Council (EPSRC), UK, project GR/S94315/01, "micro-heterogeneity and temperature effects on dynamic capillary pressure-saturation relationships for two-phase flow in porous media". Comments of two anonymous referees which have helped to improve the paper are gratefully acknowledged.

Open Access This article is distributed under the terms of the Creative Commons Attribution License which permits any use, distribution, and reproduction in any medium, provided the original author(s) and the source are credited.

\section{References}

Abidoye LK, Das DB (2014) Scale dependent dynamic capillary pressure effect for two-phase flow in porous media. Adv Water Resour. doi:10.1016/j.advwatres.2014.09.009

Akratos CS, Papaspyros JNE, Tsihrintzis VA (2008) An artificial neural network model and design equations for BOD and COD removal prediction in horizontal subsurface flow constructed wetlands. Chem Eng J 143(13):96-110

Ataie-Ashtiani B, Hassanizadeh SM, Celia MA (2002) Effects of heterogeneities on capillary pressure-saturation-relative permeability relationships. J Contam Hydrol 56(3):175-192

Ataie-Ashtiani B, Hassanizadeh SM, Oung O, Weststrate FA, Bezuijen A (2003) Numerical modelling of twophase flow in a geocentrifuge. Environ Model Softw 18(3):231-241

Baldwin JL. (1991) Proceedings of the SPE annual technical conference and exhibition, 273-286

Bear J (1972) Dynamics of fluids in porous media. Dover, New York

Boadu FK (2001) Predicting oil saturation from velocities using petrophysical models and artificial neural networks. J Pet Sci Eng 30:143-154

Bottero S, Hassanizadeh SM, Kleingeld PJ, Heimovaara TJ, (2011) Non-equilibrium capillarity effects in twophase flow through porous media at different scales. Water Resources Research 47: W10505, DOI: 10.1029/ 2011WR010887

Brooks RH, Corey AT (1964) Hydraulic properties of porous media, Hydrol. Pap. 3, Colo. State Univ., Fort Collins

Corey A (1994) Mechanics of immiscible fluids in porous media. Water Resources Publications, Highlands Ranch, Colorado

Dahle HK, Celia MA, Hassanizadeh SM (2005) Bundle-of-tubes model for calculating dynamic effects in the capillary-pressure-saturation relationship. Transp Porous Media 58(1):5-22

Das, DB, Hassanizadeh, SM, Rotter, BE, Ataie Ashtiani, B (2004) A Numerical Study of Micro-Heterogeneity Effects on Upscaled Properties of Two-Phase Flow in Porous Media. Transport in Porous Media 56: 329-350 
Das DB, Mirzaei M (2012) Dynamic effects in capillary pressure relationships for two-phase flow in porous media: experiments and numerical analyses. AIChE J 58(12):3891-3903

Das DB, Mirzaei M (2013) Experimental measurement of dynamic effect in capillary pressure relationship for two-phase flow in weakly layered porous media. AIChE J 59(5):1723-1734

Das, DB, Mirzaei, M, Widdows, N (2006) Non-uniqueness in capillary pressure-saturation-relative permeability relationships for two-phase flow in porous media. Interplay between distribution and intensity of microheterogeneity. Chemical Engineering Science 61: 6786-6803

Das DB, Gauldie R, Mirzaei M (2007) Dynamic effects in capillary pressure relationships for two-phase flow in porous media: implications of fluid properties. AIChE J 53(10):2505-2520

Das DB, Gill GS, Abidoye LK, Khudaida K (2014) A numerical study of dynamic capillary pressure effect for supercritical carbon dioxide-water flow in porous domain. AIChE J. doi:10.1002/aic.14577

Deka L, Quddus M (2014) Network-level accident-mapping: distance based pattern matching using artificial neural network. Accid Anal Prev 65:105-113

Demuth H, Beale M, Hogan M (2008) Neural network toolbox 6: user's guide, Math Works, Inc., Version 6.0.1, 1-24

Elkamel A, Karkoub K, Gharbi R (1996) A neural network prediction model of fluid displacements in porous media. Comput Chem Eng 20(1):515-520

Gharbi R, Karkoub M, Elkamel A (1995) An artificial neural network for the prediction of immiscible flood performance. Energy Fuel 9(5):894-896

Graupe D (2007) Principles of artificial neural networks advanced series on circuits and systems, vol 6. World Scientific, United Stated of America

Hanspal N, Das DB (2012) Dynamic effects on capillary pressure-saturation relationships for two-phase porous flow: implications of temperature. AIChE J 58:1951-1965

Hanspal N, Allison BA, Deka L, Das DB (2013) Artificial neural network (ANN) modeling of dynamic effects on two-phase flow in homogenous porous medial. J Hydroinf 15(2):540-554

Hassanizadeh S, Celia M, Dahle C (2002) Dynamic effect in the capillary pressure-saturation relationship and its impact on unsaturated flow. Vadose Zone J 1(1):38-40

Haykin S (1999) Neural networks - a comprehensive foundation, 2nd edn. Englewood Cliffs, NJ

Hou L, Sleep BE, Kibbey TCG (2014) The influence of unavoidable saturation averaging on the experimental measurement of dynamic capillary effects: a numerical simulation study. Adv Water Resour 66:43-51

Jain A, Varshney AK, Joshi UC (2001) Short-term water demand forecast modelling at IIT Kanpur using artificial neural networks. Water Resour Manag 15(5):299-321

Jensen J (1991) Use of the geometric average for effective permeability estimation. Math Geol 23(6):883-885

Juanes R (2009) Nonequilibrium effects in models of three-phase flow in porous media. Adv Water Resour 31: 661-673

Karimpouli S, Fathianpour N, Roohi J (2010) A new approach to improve neural networks' algorithm in permeability prediction of petroleum reservoirs using supervised committee machine neural network (SCMNN). J Pet Sci Eng 73:227-232

Kumoluyi AO, Daltaban TS (1994) Proceedings of the SPE annual western regional meeting, Long Beach, CA, 555-370

Kurtulus B, Razack M (2006) Evaluation of the ability of an artificial neural network model to simulate the inputoutput responses of a large karstic aquifer: the la rochefoucauld aquifer (Charente, France). Hydrogeol J 15(2):241-244

Levenberg K (1944) A method for the solution of certain non-linear problems in least-squares. Q Appl Math 2: $164-168$

Li D, Lake LW (1995) Scaling fluid through heterogeneous permeable media. Soc Pet Eng J 3(1):188-197

Manthey S, Hassanizadeh SM, Helmig R (2005) Macro-scale dynamic effects in homogeneous and heterogeneous porous media. Transp Porous Media 58(1-2):121-145

Mirzaei M, Das DB (2007) Dynamic effects in capillary pressure saturations relationships for two-phase flow in 3D porous media: implications of micro-heterogeneities. Chem Eng Sci 62(7):1927-1947

Mirzaei M, Das DB (2013) Experimental investigation of hysteretic dynamic effect in capillary pressuresaturation relationship for two-phase flow in porous media. AIChE J 59(10):3958-3974

Mumford KG, O’Carroll DM (2011) Dynamic effects in capillary pressure: exploring wettability and dynamic contact angle effects using bundle-of-tubes simulations. Vadose Zone J 10:1162-1172

Omole O, Falode O, Deng D (2009) Prediction of Nigerian crude oil viscosity using artificial neural network. Pet Coal 51(3):181-188

Orchard GA, Phillips (1991) Neural computation: a beginner's guide. Lawrence Erlbaum Associates, East Sussex

Parida B, Moalafhi D, Kenabatho P (2006) Forecasting runoff coefficients using ANN for water resources management: the case of notwane catchment in eastern Botswana. Phys Chem Earth A/B/C 31(15-16):928-934 
Peszynska M, Yi SY (2008) Numerical methods for unsaturated flow with dynamic capillary pressure in heterogeneous porous media. Int J Numer Anal Model 5(Special Issue):126-149

Ren L (2009) Recognition of handwriting on postal letters and parcels, school of science and technology. SIM University, Singapore

Rumelhart DE, Hinton GE, Williams RJ (1986) Learning internal representations by error propagation. In: Rumelhart DE, McLelland JL (eds) Parallel Distributed Processing: exploration in the microstructure of cognition, vol 1. MIT Press, Cambridge, pp 312-362

Sakaki T, O’Carroll DM, Illangasekare TH (2010) Direct quantification of dynamic effects in capillary pressure for drainage-wetting cycles. Vadose Zone J 9(2):424 437

Shook M, Li D, Lake WL (1992) Scaling immiscible flow through permeable media by inspectional analysis. InSitu 16(4):311-349

Smiles DE, Vachaud G, Vauclin M (1971) A test of the uniqueness of the soil moisture characteristic during transient, nonhysteretic flow in a rigid soil. Soil Sci Soc Am Proc 35:534-539

Stam J, Ziji W (1992) Modeling permeability in imperfectly layered porous media. II. A two dimensional application of block-scale permeability. Math Geol 24(8):885-891

Tabach EL, Lancelot L, Shahrour I, Najjar Y (2007) Use of artificial neural network simulation metamodelling to assess groundwater contamination in road project. Math Comput Model 45:766-776

Topp GC, Klute A, Peters DB (1967) Comparison of water content-pressure head obtained by equilibrium, steady-state, and unsteady state methods. Soil Sci Am Proc 31:312-314

Vachaud G, Vauclin M, Wakil M (1972) A study of the uniqueness of the soil moisture characteristic during desorption by vertical drainage. Soil Sci Am Proc 36:531-532

van der Heyden MJ (1996) Neural Networks and Statistics: A brief overview, International Workshop on Neural Networks, eds in van der Heyden, \& Weigl K, Proceedings Vol 1-2, VU University Press, Amsterdam

van Genuchten MT (1980) A closed-form equation for predicting the hydraulic conductivity of unsaturated soils. Soil Sci Soc Am J 44:892-898

Warren JE, Price HS (1961) Flow in heterogeneous porous media. Soc Pet Eng J 1:153-169

Werbos PE (1995) Backpropagation: basic and new developments. In: Arbib MA (ed) The handbook of brain behavior and neural networks. MIT Press, Cambridge

Zhang H, He S, Jiao C, Luan G, Mo S, Lei G (2014) Investigation of dynamic effect of capillary pressure in ultralow permeability sandstones. Indian Geotech Journal. doi:10.1007/s40098-014-0109-3 